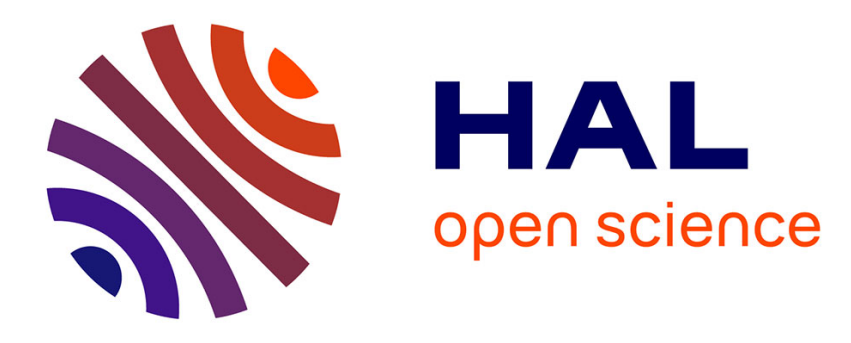

\title{
The class of representable semilattice-ordered monoids is not a variety
}

Amina Doumane

\section{To cite this version:}

Amina Doumane. The class of representable semilattice-ordered monoids is not a variety. RAMICS, Nov 2021, Marseille, France. pp.144-158. hal-03404551

\section{HAL Id: hal-03404551 https://hal.science/hal-03404551}

Submitted on 26 Oct 2021

HAL is a multi-disciplinary open access archive for the deposit and dissemination of scientific research documents, whether they are published or not. The documents may come from teaching and research institutions in France or abroad, or from public or private research centers.
L'archive ouverte pluridisciplinaire HAL, est destinée au dépôt et à la diffusion de documents scientifiques de niveau recherche, publiés ou non, émanant des établissements d'enseignement et de recherche français ou étrangers, des laboratoires publics ou privés. 


\title{
The class of representable semilattice-ordered monoids is not a variety
}

\author{
Amina Doumane \\ CNRS- ENS Lyon \\ amina.doumane@ens-lyon.fr
}

\begin{abstract}
We show a necessary and a sufficient condition for a quasivariety to be a variety. Using this, we show that the quasivariety of representable relation algebras over the signature $(\cdot, \cap, 1)$ is not a variety.
\end{abstract}

Keywords: Relations · Homomorphisms - Equational theories.

\section{Introduction}

Relations can be equipped with several natural operations: union $\cup$, intersection $\cap$, complementation ${ }^{-}$, composition $\cdot$, converse ${ }^{c}$, the empty relation 0 , the full relation $T$ and the identity relation 1 . A set of relations closed under these operations forms a proper relation algebra. We call representable relation algebras $(R R A)$ those algebras which are isomorphic to a proper relation algebra.

Representable relation algebras received a lot of attention since the seminal work of Tarski [11. However, many of these results are actually negative results. For instance, $R R A$ is not finitely axiomatizable [10] and its equational theory is undecidable [11, p88].

This motivated the investigation of the subreducts of $R R A$, that is, restrictions of $R R A$ to smaller signatures, hoping that these negative results would turn out to be positive. The subreduct that we focus on in this work is $R R A(\cdot, \cap, 1)$, the restriction of $R R A$ to the operations of composition, intersection and the identity relation, also known as the class of representable semi-lattice ordered monoids. It was deeply studied in [2] and 8. For example, its equational theory is decidable [1] but not finitely axiomatizable [5].

Despite all the negative results about $R R A$, it enjoys an important positive result: it is a variety [12. That is to say, membership in $R R A$ can be characterized by (a possibly infinite) set of equations. It was then natural to ask whether this result holds also for its subreducts.

The positive subreducts 1 of $R R A$ are known to be quasi-varieties, i. e., membership can be characterized by (a possibly infinite) set of Horn clauses [2] However, some of them are not varieties [1, Thm.6].

\footnotetext{
1 Those subreducts that do not use negation.
} 
Despite an attempt in [8, the status of $R R A(\cdot, \cap, 1)$ is not known ${ }^{2}$ In this paper, we show that $R R A(\cdot, \cap, 1)$ is also not a variety.

We use a technique similar to [1]. Actually, we abstract their argument in a more general setting, then we apply it to our particular case. More precisely, we proceed in two steps.

First, we give a necessary and sufficient condition for a quasivariety to be a variety. In words, this condition says that a quasivariety $\mathcal{C}$ is a variety if and only if, for every Horn clause $(\mathcal{H} \Rightarrow t=u)^{3}$ which is valid in $\mathcal{C}$, its conclusion $(t=u)$ can be "deduced" from its hypothesis $\mathcal{H}$.

This gives us a strategy to show that $R R A(\cdot, \cap, 1)$ is not a variety. It is "enough" to find a Horn clause which is valid in $R R A(\cdot, \cap, 1)$ but whose conclusion cannot be deduced from its hypothesis. Of course, the difficulty here is to guess this Horn clause and to show that indeed its conclusion is not provable from its hypothesis. For this purpose, we rely on graph theoretical tools and intuitions coming from a well established graph characterization of the equational theory of $\operatorname{RRA}(\cdot, \cap, 1)$ [1].

Outline. In Sec. 2, we define varieties, quasi-varieties and their equational and Horn theories. Then we introduce the quasivariety $R R A(\cdot, \cap, 1)$ and the graph characterization of its equational and Horn theories. We show in Sec. 3 the necessary and sufficient condition for a quasivariety to be a variety. Building on this, we show in Sec. 4 that $R R A(\cdot, \cap, 1)$ is not a variety.

\section{Preliminaries}

\subsection{Algebras, varieties and quasi-varieties}

Algebras. A signature is a pair $\mathcal{S}=(O, a r)$ where $O$ is a set of operations, and ar $: O \rightarrow \omega$ is a function assigning to each operation an integer called its arity. An algebra over $\mathcal{S}$ consists of a set $D$ called its domain, and for each operation $o$ of $\mathcal{S}$ with arity $n$, a function $f_{o}: D^{n} \rightarrow D$.

Equations and Horn clauses. We fix in the rest of the paper a set $X$ of variables. Terms over a signature $\mathcal{S}=(O, a r)$ are generated by the following syntax:

$$
t:=x \mid o\left(t_{1}, \ldots, t_{n}\right) \quad x \in X, o \in O \text { and } n=\operatorname{ar}(o)
$$

We denote the set of terms by $\mathcal{T}_{X}(\mathcal{S})$, but if the signature and the set of variables are clear from the context, we denote it simply $\mathcal{T}$. An equation is a pair of terms that we usually write $(t=u)$. A Horn clause consists of a finite set of equations

\footnotetext{
${ }^{2}$ The proof that $R R A(\cdot, \cap, 1)$ is not a variety in 8 relies on the claim that the equational theory of $R R A(\cdot, \cap, 1)$ is finitely axiomatizable [2], which turns out to be wrong, see 9 and 5

${ }^{3}$ Here, $\mathcal{H}$ is a conjunction of equations called the hypothesis, the equation $(t=u)$ is the conclusion.
} 
$\mathcal{H}$ called its hypothesis and an equation called its conclusion, we usually write it like this $(\mathcal{H} \Rightarrow t=u)$. An equation can be seen as a Horn clause with an empty set of hypothesis.

Truth. Let $\mathcal{A}$ be an algebra over a signature $\mathcal{S}$, and let $D$ be the domain of $\mathcal{A}$. An interpretation is a function $\sigma: X \rightarrow D$ mapping variables into elements of $D$. We can extend $\sigma$ to all terms $\sigma: \mathcal{T} \rightarrow D$, by interpreting the operations of $\mathcal{S}$ as the corresponding functions of $\mathcal{A}$.

Let $\sigma$ be an interpretation as above. An equation $(t=u)$ is true in $\mathcal{A}$ under $\sigma$, noted

$$
\mathcal{A}, \sigma \models(t=u)
$$

if $\sigma(t)=\sigma(u)$. A set of equations $\mathcal{H}$ are true in $\mathcal{A}$ under $\sigma$, noted

$$
\mathcal{A}, \sigma \models \mathcal{H}
$$

if this is the case for every equation in $\mathcal{H}$. A Horn clause

$$
\varphi:=(\mathcal{H} \Rightarrow t=u)
$$

is true in $\mathcal{A}$ under $\sigma$, noted

$$
\mathcal{A}, \sigma \models \varphi
$$

if either $\mathcal{A}, \sigma \not \forall \mathcal{H}$ or $\mathcal{A}, \sigma=(t=u)$. We say that $\varphi$ is true in $\mathcal{A}$, noted $\mathcal{A}=\varphi$, if $\varphi$ is true in $\mathcal{A}$ under all possible interpretations.

(Quasi-) Varieties. We have introduced individual algebras, now we focus on classes of algebras. Let $\mathcal{C}$ be a class of algebras over a signature $\mathcal{S}$. We say that an equation or a Horn clause $\varphi$ is valid in $\mathcal{C}$, and write $\mathcal{C}=\varphi$ if $\varphi$ is true in every algebra of $\mathcal{C}$. The equational theory (resp. Horn theory) of $\mathcal{C}$ denoted $\mathcal{E} q(\mathcal{C})$ (resp. $\mathcal{H} \operatorname{orn}(\mathcal{C})$ ) is the set of equations (resp. Horn clauses) which are valid in $\mathcal{C}$.

Let $\mathcal{C}$ be a class of algebras over $\mathcal{S}$ and let $E$ be a set of equations or Horn clauses. We say that $E$ axiomatizes $\mathcal{C}$ if for every algebra $\mathcal{A}$ over $\mathcal{S}$ :

$$
\mathcal{A} \in \mathcal{C} \quad \text { iff } \quad \mathcal{A}=E
$$

We say that a class of algebras is a variety (resp. quasivariety) if it can be axiomatized by a set of equations (resp. Horn clauses).

Remark 1. Note that if $\mathcal{C}$ is a variety (resp. quasivariety), then $\mathcal{C}$ is axiomatized by its equational (resp. Horn) theory.

\subsection{Representable relation algebras}

In this paper, we focus on the signature whose set of operations is $\{\cdot, \cap, 1\}$, the operations · and $\cap$ being of arity 2 and the operation 1 being of arity 0 . We will write this signature $(\cdot, \cap, 1)$. To lighten notations of terms over this signature, we often write $t u$ for $t \cdot u$, and assign priorities to operations so that $a b \cap c$ parses as $(a \cdot b) \cap c$. 
A proper relation algebra is an algebra over $(\cdot, \cap, 1)$ whose universe $U$ is a set of relations, that is $U \subseteq \mathcal{P}(B \times B)$, where $B$ is a base set, the operations - and $\cap$ are respectively the composition and intersection of relations, and 1 is the identity relation over $B$. A representable relation algebra is an algebra over $(\cdot, \cap, 1)$ which is isomorphic to a proper relation algebra; we denote their set by $\operatorname{RRA}(\cdot, \cap, 1)$.

The class of algebras $R R A(\cdot, \cap, 1)$ forms a quasivariety [2, p.2]. The goal of this paper is to show that we cannot say more: $R R A(\cdot, \cap, 1)$ is not a variety.

In $R R A(\cdot, \cap, 1)$, it will be convenient to work with inequations instead of equations. An inequation is a pair of terms written as $(t \geq u)$, which is a shortcut for the equation $(t \cap u=u)$. Similarly, we will work with Horn clauses which use inequations in their hypothesis and conclusions instead of equations. By definition, every inequation is an equation, and conversely every equation $(t=u)$ is equivalent to the two equations $(t \geq u)$ and $(u \geq t)$. Similarly, every Horn clause is equivalent to a set of Horn clauses using inequations. In the sequel, when dealing with relation algebras, we will mostly work with inequations and Horn clauses using inequations. We call the inequational (resp. Horn) theory of relations the set of inequations (resp. Horn clauses using inequations) which are valid in $R R A(\cdot, \cap, 1)$.

\subsection{Characterization of the inequational and Horn theory of $\operatorname{RR} A(\cdot, \cap, 1)$}

Graphs. A 2-pointed labeled graph is a tuple $(V, E, \iota, o)$ where $V$ is a set of vertices, $E \subseteq V \times A \times V$ is a set of edges, $A$ is a set of labels and $\iota$ and $o$ are two distinguished vertices called the input and output. We simply call them graphs in the sequel; we depict them as expected, with unlabeled ingoing and outgoing arrows to denote the input and the output, respectively. We denote by $\mathcal{G}$ the set of all graphs.

We define the operations · and $\cap$ on graphs as follows:

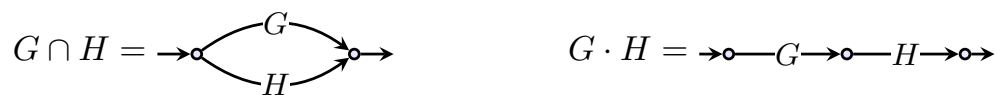

We associate to every term $t \in \mathcal{T}$ a graph $\mathcal{G}(t)$ called the graph of $t$, by letting:

$$
\mathcal{G}(1)=\longrightarrow \text { - } \quad \mathcal{G}(x)=\longrightarrow \stackrel{x \rightarrow 0 \longrightarrow}{\longrightarrow} \quad(x \in X)
$$

and by interpreting the operations · and $\cap$ on graphs as above.

Example 1. The graphs of the terms $x y \cap x z$ and $x y \cap 1$ are respectively the following:
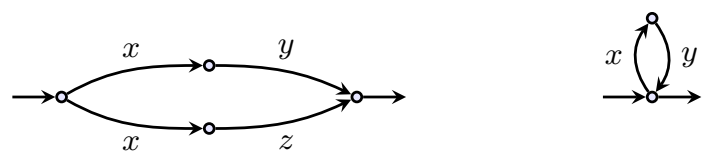
Graph terms. A graph term is a graph which is the image of some term, and we denote by $\mathcal{G}_{t}$ the set of graph terms. Not every graph is a graph term. For instance, graph terms do not contain the graph $K_{4}$, the complete graph with four vertices (see Fig. 1 (a)), as a minor [3]. Graph terms also do not contain back patterns [5], which we recall below.

Definition 1 (Back pattern). A back pattern in a graph is a pair of distinct nodes $m, n$ together with three directed paths: $\pi$ from the input to $m, \kappa$ from $n$ to $m$, and $\rho$ from $n$ to the output, such that $\pi$ and $\kappa$ intersect exactly on $m$ and $\kappa$ and $\rho$ intersect exactly on $n$.

Such a back pattern can be depicted as follows: $\iota \stackrel{\pi}{\longrightarrow} m \stackrel{\kappa}{\stackrel{\kappa}{\longrightarrow}} \stackrel{\rho}{\longrightarrow} 0$.

Proposition 1 ([3, Cor.27],[5, Prop. 12]). Graph terms do not contain back patterns, nor $K_{4}$ as a minor.

Example 2. The graph of Fig. 1(b) is not a graph term, no matter how we label or orient the edges, because it contains $K_{4}$ as a minor. Indeed, if we remove the green edge and contract one of the two blue edges, we obtain $K_{4}$. The graph of Fig. 1. (c) is not a graph term, no matter how we label its edges, because it contains a back pattern, colored in red.

(a)



(b)

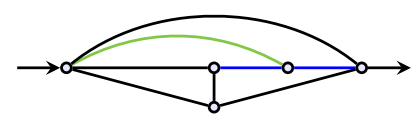

(c)

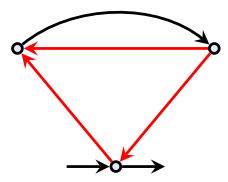

Fig. 1. (a) The graph $K_{4}$. (b) A graph containing $K_{4}$ as a minor. (c) A graph with a back pattern.

Homomorphisms. Given two graphs $G=\langle V, E, \iota, o\rangle$ and $G^{\prime}=\left\langle V^{\prime}, E^{\prime}, \iota^{\prime}, o^{\prime}\right\rangle$, a homomorphism $h: G \rightarrow G^{\prime}$ is a mapping from $V \rightarrow V^{\prime}$ that preserves labeled edges, ie. if $(x, a, y) \in E$ then $(h(x), a, h(y)) \in E^{\prime}$, and preserves input and output, ie. $h(\iota)=\iota^{\prime}$ and $h(o)=o^{\prime}$. We say that the homomorphism $h$ identifies the vertices $x$ and $y$ if $h(x)=h(y)$. We write $G \triangleright G^{\prime}$ if there exists a graph homomorphism from $G$ to $G^{\prime}$.

Characterizing the inequational theory of relations. The inequational theory of $R R A(\cdot, \cap, 1)$ can be characterized using graphs and homomorphisms as follows.

${ }^{4}$ A graph $G$ is a minor of a graph $H$ if $G$ can be obtained from $H$ by deleting some edges and vertices and contracting some edges. 
Theorem 1 ([1, Thm. 1], [6, p. 208]). For all terms $t, u$,

$$
R R A(\cdot, \cap, 1) \models(t \geq u) \quad \text { iff } \quad \mathcal{G}(t) \triangleright \mathcal{G}(u)
$$

Example 3. The validity of the inequation $x y \cap x z \geq x(y \cap z)$ is witnessed by the following homomorphism:

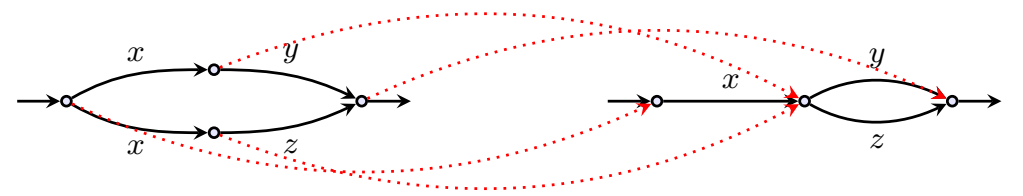

Characterizing the Horn theory of relations. A graph context is a graph with a distinguished edge labeled by a special letter $\bullet$, called its hole. If $G$ is a graph and $C$ a graph context, then $C[G]$ is the graph obtained by "plugging $G$ in the hole" of $C$, that is, $C[G]$ is the graph obtained as the disjoint union of $G$ and $C$, where we identify the input (resp. output) of $G$ with the input (resp. output) of the edge labeled by $\bullet$ in $C$, and we remove the edge of $C$ labeled $\bullet$. Here is an example:


Definition 2 (The relation $\triangleright_{\mathcal{H}}$ ). Let $\mathcal{H}$ be a set of inequations. We define the relation $>_{\mathcal{H}}$ on graphs as follows. We set $G>_{\mathcal{H}} H$ if there exists a graph context $C$ and an inequation $(t \geq u) \in \mathcal{H}$ such that:

$$
G=C[\mathcal{G}(t)] \quad \text { and } \quad H=C[\mathcal{G}(u)]
$$

We define $\triangleright_{\mathcal{H}}$ as the transitive closure of $\triangleright \cup>_{\mathcal{H}}$.

In the definition above, the graphs $G, H$ and $C$ are not necessarily graph terms.

Theorem 2 ([4, Thm. 12]). For all terms t, $u$ and set of inequations $\mathcal{H}$, we have:

$$
R R A(\cdot, \cap, 1) \models(\mathcal{H} \Rightarrow t \geq u) \quad \text { iff } \quad \mathcal{G}(t) \triangleright_{\mathcal{H}} \mathcal{G}(u)
$$

Hence, in order to show that a Horn clause $(\mathcal{H} \Rightarrow t \geq u)$ is valid, we need to find a sequence of graphs $G_{0}, \ldots, G_{n}$ such that $G_{0}=\mathcal{G}(t), G_{n}=\mathcal{G}(u)$ and for every $i \in[0, n-1]$ the graphs $G_{i}$ and $G_{i+1}$ are either related by homomorphism or by the relation $>_{\mathcal{H}}$. We say that this sequence witnesses the validity of this Horn sentence.

Example 4. The validity of the following Horn clause

$$
x y \geq x \Rightarrow x y y \cap x z \geq x(y \cap z)
$$

is witnessed by the following sequence: 




The picture should be read from left to right. First, we identified in the graph of $x y y \cap x z$ a context (in red) and the graph of $x y$ (in green). As allowed by the hypothesis $x y \geq x$, we replaced the graph of $x y$ by that of $x$, this is a $>_{\mathcal{H}}$ step. Finally we apply a homomorphism to get the graph of $x(y \cap z)$.

\section{When is a quasivariety a variety?}

In the rest of this section we fix a signature $\mathcal{S}$ and a set of variables $X$. Algebras will be over $\mathcal{S}$ and terms over $\mathcal{S}$ and $X$. We omit the mention of the signature as it is clear from the context. In the following we give a necessary and sufficient condition for a quasivariety to be a variety.

A context is a term with a unique occurrence of the special variable $\bullet$ called its hole. If $C$ is a context and $t$ a term, then $C[t]$ denotes the substitution of the variable $\bullet$ by $t$ in $C$.

Definition 3. Let $\mathcal{C}$ be a class of algebras and $\mathcal{H}$ be a set of equations. We define the relation $\underset{\mathcal{H}}{\overline{\mathcal{H}}}$ on terms as follows. We set $(t \underset{\mathcal{H}}{=} u)$ if there exists a term context $C$ and an equation $\left(t^{\prime}=u^{\prime}\right) \in \mathcal{H}$ such that:

$$
t=C\left[t^{\prime}\right] \quad \text { and } \quad u=C\left[u^{\prime}\right]
$$

We define the relation $\underset{\mathcal{H}, \mathcal{C}}{=}$ as the transitive closure of the relation $(\mathcal{E} q(\mathcal{C}) \cup \underset{\mathcal{H}}{=})$ $i$. e., the union of the equational theory of $\mathcal{C}$ and the relation $\overline{\overline{\mathcal{H}}}$.

If $(t \underset{\mathcal{H}, \mathcal{C}}{=} u)$ we say that the equation $(t=u)$ is a consequence of the hypothesis $\mathcal{H}$ in the algebras $\mathcal{C}$.

In words, we have $(t \underset{\mathcal{H}, \mathcal{C}}{=} u)$ if there exists a sequence of terms $t_{0}, \ldots, t_{n}$ such that $t_{0}=t, t_{n}=u$ and for every $i \in[0, n-1]$ the equation $t_{i}=t_{i+1}$ is either valid in $\mathcal{C}$ or is obtained as the application of a context to an equation of $\mathcal{H}$. We call such sequence a witness of $(t \underset{\mathcal{H}, \mathcal{C}}{=} u)$.

Remark 2. An alternative definition for $\underset{\mathcal{H}, \mathcal{C}}{=}$ would have been to use a proof system which uses the equations of $\mathcal{H}$ and $\mathcal{E} q(\mathcal{C})$ as axioms.

A congruence on terms is an equivalence relation on terms that is stable under contexts.

Lemma 1. The relation $\underset{\mathcal{H}, \mathcal{C}}{=}$ is a congruence on terms. 
Proof. The relation $\underset{\mathcal{H}, \mathcal{C}}{=}$ is clearly an equivalence relation. Let us show that it is stable under contexts. We proceed by induction on the length of a witness sequence for $(t \underset{\mathcal{H}, \mathcal{C}}{=} u)$. If $(t, u) \in \mathcal{E} q(\mathcal{C})$ then we have also $(C[t], C[u]) \in \mathcal{E} q(\mathcal{C})$ because $\mathcal{E} q(\mathcal{C})$ is a congruence. If $(t \underset{\mathcal{H}}{\overline{\mathcal{H}}} u)$ then $(C[t] \underset{\mathcal{\mathcal { H }}}{\overline{=}} C[u])$, because the composition of two contexts is also a context. The inductive step is immediate.

The following theorem says that a quasivariety $\mathcal{C}$ is a variety if and only if the conclusion of every valid Horn clause is a consequence of its hypothesis in $\mathcal{C}$.

Theorem 3. A quasivariety $\mathcal{C}$ is a variety if and only if the following holds

$$
(\mathcal{H} \Rightarrow t=u) \in \mathcal{H} \operatorname{orn}(\mathcal{C}) \quad \Rightarrow \quad t \underset{\mathcal{H}, \mathcal{C}}{=} u
$$

Remark 3. Note that the reverse implication of $(\dagger)$ is true, regardless of whether $\mathcal{C}$ is a variety or not.

Proof. $(\Rightarrow)$ Suppose that $\mathcal{C}$ is a variety and let $(\mathcal{H} \Rightarrow t=u)$ be a Horn clause valid in $\mathcal{C}$. Let $\mathcal{T}_{\mathcal{H}, \mathcal{C}}$ be the algebra of terms quotiented by the congruence $\underset{\mathcal{H}, \mathcal{C}}{=}$. The algebra $\mathcal{T}_{\mathcal{H}, \mathcal{C}}$ satisfies all the equations in $\mathcal{E} q(\mathcal{C})$ because $\underset{\mathcal{H}, \mathcal{C}}{=}$ contains $\mathcal{E} q(\mathcal{C})$. Since $\mathcal{C}$ is a variety, we have that $\mathcal{T}_{\mathcal{H}, \mathcal{C}} \in \mathcal{C}$.

Consider the interpretation $\sigma$ which assigns to every letter its equivalence class w.r.t. the relation $\underset{\mathcal{H}, \mathcal{C}}{=}$. We have that

$$
\mathcal{T}_{\mathcal{H}, \mathcal{C}}, \sigma \models \mathcal{H}
$$

because the relation $\underset{\mathcal{H}, \mathcal{C}}{=}$ contains $\mathcal{H}$. Since $(\mathcal{H} \Rightarrow t=u) \in \mathcal{H}$ orn $(\mathcal{C})$, we have that

$$
\mathcal{T}_{\mathcal{H}, \mathcal{C}}, \sigma \models(t=u)
$$

which is the same thing as $(t \underset{\mathcal{H}, \mathcal{C}}{=} u)$.

$(\Leftarrow)$ Suppose that $(\dagger)$ holds and let us show that $\mathcal{C}$ is a variety. Let $\mathcal{A}$ be an algebra satisfying all the equations in $\mathcal{E} q(\mathcal{C})$, we show that $\mathcal{A}$ is an algebra in $\mathcal{C}$. As $\mathcal{C}$ is a quasivariety, it is enough to show that $\mathcal{A}$ satisfies all Horn clauses valid in $\mathcal{C}$. Let $(\mathcal{H} \Rightarrow t=u)$ be such Horn clause and let $\sigma$ be an interpretation such that $\mathcal{A}, \sigma \models \mathcal{H}$. By $(\dagger)$, we have that $(t \underset{\mathcal{H}, \mathcal{C}}{=} u)$. We can show by a simple induction on the length of a sequence justifying $(t \underset{\mathcal{H}, \mathcal{C}}{=} u)$ that we have $\mathcal{A}, \sigma \models(t=u)$. This concludes the proof.

\section{The quasivariety $R R A(\cdot, \cap, 1)$ is not a variety}

Let us first specify Theorem 3 for the quasivariety $R R A(\cdot, \cap, 1)$. For that, we define below the relation $\boldsymbol{\mathcal { H }}_{\mathcal{H}}$ on graph terms. Recall that graph terms are those graphs coming from terms, and that we denote their set by $\mathcal{G}_{t}$. 
Definition 4 (The relation $\nabla_{\mathcal{H}}$ ). Let $\mathcal{H}$ be a set of inequations. We define the relation $\succ_{\mathcal{H}}$ on graph terms as follows. For $G, H \in \mathcal{G}_{t}$, we set $G \succ_{\mathcal{H}} H$ if there exists a context $C \in \mathcal{G}_{t}$ and an inequation $(t \geq u) \in \mathcal{H}$ such that:

$$
G=C[\mathcal{G}(t)] \quad \text { and } \quad H=C[\mathcal{G}(u)]
$$

We define the relation $\boldsymbol{}_{\mathcal{H}} \subseteq \mathcal{G}_{t} \times \mathcal{G}_{t}$ as the transitive closure of the relation $\triangleright \cup \succ_{\mathcal{H}}$.

In words, $G \boldsymbol{}_{\mathcal{H}} H$ if there exists a sequence of graph terms $G_{0}, \ldots, G_{n}$ such that $G_{0}=G, G_{n}=H$ and for every $i \in[0, n-1]$ the graphs $G_{i}$ and $G_{i+1}$ are either related by homomorphism or by the relation $\succ_{\mathcal{H}}$. We say that this sequence is a witness for $G \boldsymbol{}_{\mathcal{H}} H$. The relation $\boldsymbol{}_{\mathcal{H}}$ should not be confused with the relation $\triangleright_{\mathcal{H}}$. Indeed, for the latter, the graphs of a witness sequence may not be graph terms.

Using Theorem 1 we can adapt Theorem 3 to get the following version for relation algebras.

Theorem 4. If $R R A(\cdot, \cap, 1)$ is a variety then the following holds

$$
R R A(\cdot, \cap, 1) \models(\mathcal{H} \Rightarrow t \geq u) \quad \Rightarrow \quad \mathcal{G}(t) \triangleright_{\mathcal{H}} \mathcal{G}(u)
$$

Proof. Suppose that $R R A(\cdot, \cap, 1)$ is a variety and let $(\mathcal{H} \Rightarrow t \geq u)$ be a Horn clause valid in $R R A(\cdot, \cap, 1)$. To simplify notations suppose that $\mathcal{H}=\{v \geq w\}$. The general case can be treated similarly. Note that the Horn clause above is a shortcut for

$$
(v \cap w=w) \Rightarrow(t \cap u=u)
$$

By Theorem 3. there is a sequence of terms $t_{0}, \ldots, t_{n}$ such that $t_{0}=(t \cap u), t_{n}=u$ and for every $i \in[0, n-1]$ the equation $\left(t_{i}=t_{i+1}\right)$ is either valid in $R R A(\cdot, \cap, 1)$ or is obtained as an application of the hypothesis $(v \cap w=w)$ under some context.

Let us show that $\mathcal{G}(t) \nabla_{\mathcal{H}} \mathcal{G}(u)$. We have that $\mathcal{G}(t) \triangleright \mathcal{G}(t \cap u)$, so we only need to show that $\mathcal{G}(t \cap u)>_{\mathcal{H}} \mathcal{G}(u)$. For that, we exploit the sequence above. Note that if $\left(t_{i}=t_{i+1}\right)$ is valid in $\operatorname{RRA}(\cdot, \cap, 1)$, then $\mathcal{G}\left(t_{i}\right) \triangleright \mathcal{G}\left(t_{i+1}\right)$ by Theorem 1 If $\left(t_{i}=t_{i+1}\right)$ is obtained as an application of the hypothesis $(v \cap w=w)$ under some context $C$, we can distinguish two cases.

- Either $t_{i}=C[w]$ and $t_{i+1}=C[v \cap w]$. In this case we have $\mathcal{G}\left(t_{i}\right) \triangleright \mathcal{G}\left(t_{i+1}\right)$ because $\mathcal{G}(w) \triangleright \mathcal{G}(v \cap w)$.

- Or $t_{i}=C[v \cap w]$ and $t_{i+1}=C[w]$. In this case we show that $\mathcal{G}\left(t_{i}\right){ }_{\mathcal{H}} \mathcal{G}\left(t_{i+1}\right)$. Indeed, Let $C^{\prime}$ be the graph context $\mathcal{G}(C[\bullet \cap w])$. We have that

$$
\mathcal{G}\left(t_{i}\right)=C^{\prime}[\mathcal{G}(v)]>_{\mathcal{H}} C^{\prime}[\mathcal{G}(w)]=\mathcal{G}(C[w \cap w]) \triangleright \mathcal{G}(C[w])=\mathcal{G}\left(t_{i+1}\right)
$$

This ends the proof.

This gives us a strategy to show that $R R A(\cdot, \cap, 1)$ is not a variety: it is enough to find a Horn clause $(\mathcal{H} \Rightarrow t \geq u)$ which is valid for relations, but for which $\mathcal{G}(t){ }_{\mathcal{H}} \mathcal{G}(u)$. We explicit such counter-example below. 
Definition 5 (The counter-example). Let $\mathbb{X}=\{x, y, z, a, b, c\}$ be a set of variables. We define the terms $\eta$ and $\rho$ and the set of equations $\mathcal{K}$ over $\mathbb{X}$ as follows:

$\eta:=x y(z \cap a(b y a \cap 1) b c(d x c \cap 1) d) \cap 1 \quad \rho:=x y z \cap 1 \quad \mathcal{K}:=\{a b \geq z x, c d \geq y z\}$

We show that $(\mathcal{K} \Rightarrow \eta \geq \rho)$ is indeed a counter-example. This is Prop. 2 below.

Proposition 2. We have that:

$$
\operatorname{RRA}(\cdot, \cap, 1) \models(\mathcal{K} \Rightarrow \eta \geq \rho) \quad \text { but } \quad \mathcal{G}(\eta){ }_{\mathcal{K}} \mathcal{G}(\rho) \text {. }
$$

Corollary 1. The quasivariety $R R A(\cdot, \cap, 1)$ is not a variety.

Proof (of the first part of Prop. 2). To prove that $(\mathcal{K} \Rightarrow \eta \geq \rho)$ is a valid Horn clause, we can either proceed by a direct but boring proof, or use Theorem 2 . We choose the second option, and show that $\mathcal{G}(\eta) \triangleright_{\mathcal{K}} \mathcal{G}(\rho)$. In Fig. 2, we explicit a sequence witnessing that $\mathcal{G}(\eta) \triangleright_{\mathcal{K}} \mathcal{G}(\rho)$. It should be read from top left, then down, then top right: we start by applying a homomorphism to the graph $\mathcal{G}(\eta)$; the nodes which are identified by the homomorphism are linked by the dotted lines. Then we apply the relation $>_{\mathcal{K}}$ : the graph of $a b$ is colored in green, we replace it by $z x$ as allowed by the set $\mathcal{K}$. We apply again $>_{\mathcal{K}}$, this time by replacing the graph of $c d$ by the graph of $y z$. We finally apply a homomorphism to get $\mathcal{G}(\rho)$.


Fig. 2. A sequence witnessing that $\mathcal{G}(\eta) \triangleright_{\mathcal{K}} \mathcal{G}(\rho)$. 
Note that the intermediary graphs of the witness sequence of Fig. 2 are not graph terms. For example, the second graph from the right contains a back pattern (Def. 1), colored in red.

Let us now explicit our strategy to prove that $\mathcal{G}(\eta){ }_{\mathcal{K}} \mathcal{G}(\rho)$. First, let us remark that $\mathcal{G}(\eta) \not \mathcal{G}(\rho)$. Indeed, no homomorphism can preserve the edges labeled $a, b, c$ and $d$. We will show that under some constraints on $G$ and $H$, we have the following result:

$$
\text { If } G \triangleright_{\mathcal{K}} H \quad \text { and } \quad \mathcal{G}(\eta) \triangleright G \quad \text { then } \quad \mathcal{G}(\eta) \triangleright H \quad \text { (Lem. } 4 \text { ) }
$$

By taking $G$ and $H$ to be respectively $\mathcal{G}(\eta)$ and $\mathcal{G}(\rho)$ in this result, and using the remark above, we can show by contradiction that $\mathcal{G}(\eta){ }_{\mathcal{K}} \mathcal{G}(\rho)$.

As said before, Lem. 4 is true under some constraints on the graphs $G$ and $H$. More precisely, it is true when they do not contain some specific graphs called persistent patterns as sub-graphs. In the following we define these persistent patterns and show Lem. 4

Definition 6 (Persistent patterns). Persistent patterns are the graphs of Fig. 鸟.

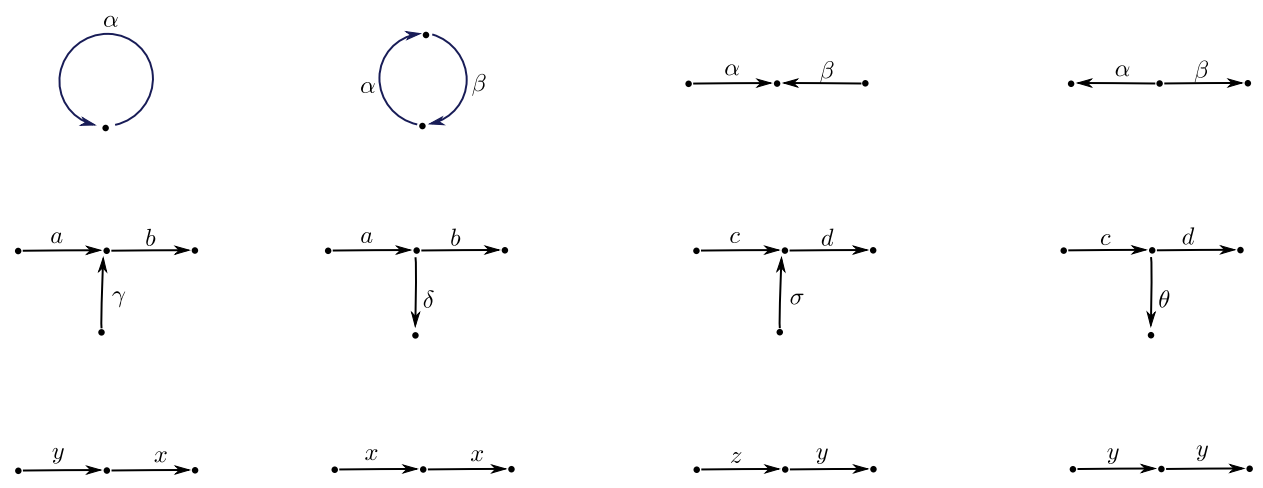

Fig. 3. Persistent patterns. The vertices of these graphs may not be distinct. All labels belong to $\mathbb{X}$, the variables used in the counter example (Def. 5) with the following constraints: $\alpha \neq \beta, \gamma \neq a, \delta \neq b, \sigma \neq c$ and $\theta \neq d$.

Persistent patterns are called so because they satisfy the following property, whose proof is a simple case distinction.

Lemma 2. If $G \bullet_{\mathcal{K}} H$ and $G$ contains a persistent pattern, then $H$ also contains a persistent pattern.

For convenience, we label the nodes of $\mathcal{G}(\eta)$ as in Fig. 4 . We say that a vertex of 


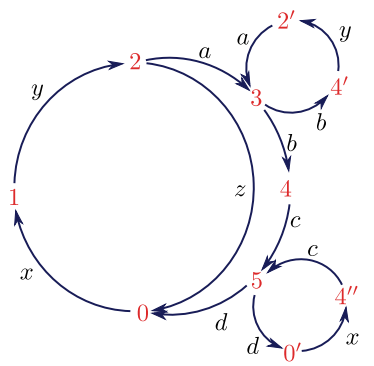

Fig. 4. Labeling the vertices of $\mathcal{G}(\eta)$.

$\mathcal{G}(\eta)$ is tagged by an integer $i$ if its label contains $i$. For instance, the vertex labeled by $2^{\prime}$ is tagged by the integer 2 . To prove Lem. 4, we need the following result, which says that a homomorphism from $\mathcal{G}(\eta)$ to any graph term not containing a persistent pattern cannot identify vertices tagged by distinct integers.

Lemma 3. Let $G$ be a graph term not containing a persistent pattern, and let $h: \mathcal{G}(\eta) \rightarrow G$ be a homomorphism from $\mathcal{G}(\eta)$ to $G$. The homomorphism $h$ cannot identify two vertices tagged by distinct integers.

Note however that $h$ may identify two vertices tagged by the same integer, for instance the vertices labeled by 2 and $2^{\prime}$.

Proof (of Lem. 3). We show that if $h$ identifies two nodes tagged by distinct integers, then $G$ contains necessarily a persistent pattern, a back pattern or $K_{4}$ as a minor. Fig. 5 shows the persistent patterns that appear if we identify nodes with distinct tags. The gray cells are symmetric and the white cells correspond to vertices tagged by the same integer. To complete the proof, we need to prove that the vertex tagged by 1 cannot be identified by a vertex tagged by 4 , these cases correspond to the cells $(\star),(\dagger)$ and $(\ddagger)$. We show that if we do such identification, we create a back pattern or $K_{4}$ as a minor.

$(\star)$ If we identify the vertices labeled 1 and 4 we create the following back pattern, where the vertex labeled $(1,4)$ is the image of 1 and 4 by $h$. It is a back pattern because its vertices are pairwise distinct. Indeed, if its vertices were not pairwise distinct, then we would create one of the patterns treated in Fig. 5 .

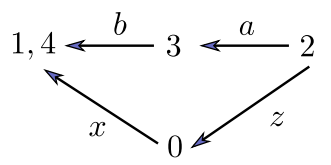

$(\dagger)$ If we identify the vertices labeled 1 and $4^{\prime}$ we create the same back pattern.

( ) If we identify the vertices labeled 1 and $4^{\prime \prime}$ we distinguish two cases. Either the vertices 0 and $0^{\prime}$ are not identified by $h$, and in this case we create this back pattern: 




Otherwise, 0 and $0^{\prime}$ are identified by $h$. We can suppose that 1 and 4 are not identified by $h$ (otherwise, this case was treated by $(\star)$ ). In this case, we obtain the following graph as a subgraph, which contains the graph $K_{4}$ as a minor:

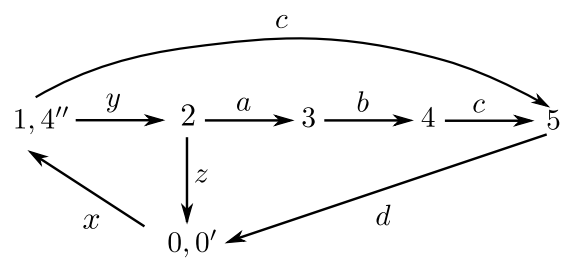

Lemma 4. Let $G, H$ be two graphs and suppose that $H$ does not contain a persistent pattern. We have that:

$$
\text { If } G \triangleright_{\mathcal{K}} H \quad \text { and } \quad \mathcal{G}(\eta) \triangleright G \quad \text { then } \quad \mathcal{G}(\eta) \triangleright H
$$

Proof. We proceed by induction on length of the sequence witnessing that $G \triangleright_{\mathcal{K}}$ $H$. Note that since $H$ does not contain a persistent pattern, all the graphs of the witness sequence do not contain a persistent pattern as well thanks to Lem. 2 .

The inductive step is easy, the interesting part are the base cases. The first one is when $G \triangleright H$, and we have clearly that $\mathcal{G}(\eta) \triangleright G$ implies that $\mathcal{G}(\eta) \triangleright H$ because we can compose these two homomorphisms. Now suppose that $G \succ_{\mathcal{K}} H$. There are two cases to consider: we have either used the inequation $a b \geq z x$ or $c d \geq y z$ to justify $G \succ_{\mathcal{K}} H$. Suppose that we are in the first case, i. e., there exists a context $C \in \mathcal{G}_{t}$ such that:

$$
G=C[\mathcal{G}(a b)] \quad \text { and } \quad H=C[\mathcal{G}(z x)]
$$

Let $h$ be a homomorphism from $\mathcal{G}(\eta)$ to $G$. Our goal is to show that the image of $h$ lies in $C$. If we do so, we can easily prove that $\mathcal{G}(\eta) \triangleright H$ because $h$ can also be used to map $\mathcal{G}(\eta)$ to $H$.

Let $m$ be the inner vertex of the graph $\mathcal{G}(a b)$, that is the vertex distinct from the input and output. We show that no vertex of $\mathcal{G}(\eta)$ can be mapped by $h$ to $m$. Suppose for contradiction that there exits a vertex of $\mathcal{G}(\eta)$ whose image is $m$. This vertex is necessarily the vertex 3 (see Fig. (4), and we have necessarily that $h(2)=h\left(2^{\prime}\right)$ and $h(4)=h\left(4^{\prime}\right)$. But this creates the following back-pattern in $G$, where as usual a node labeled by two integers is the common image of the corresponding vertices of $\mathcal{G}(\eta)$ :

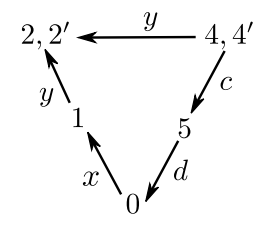




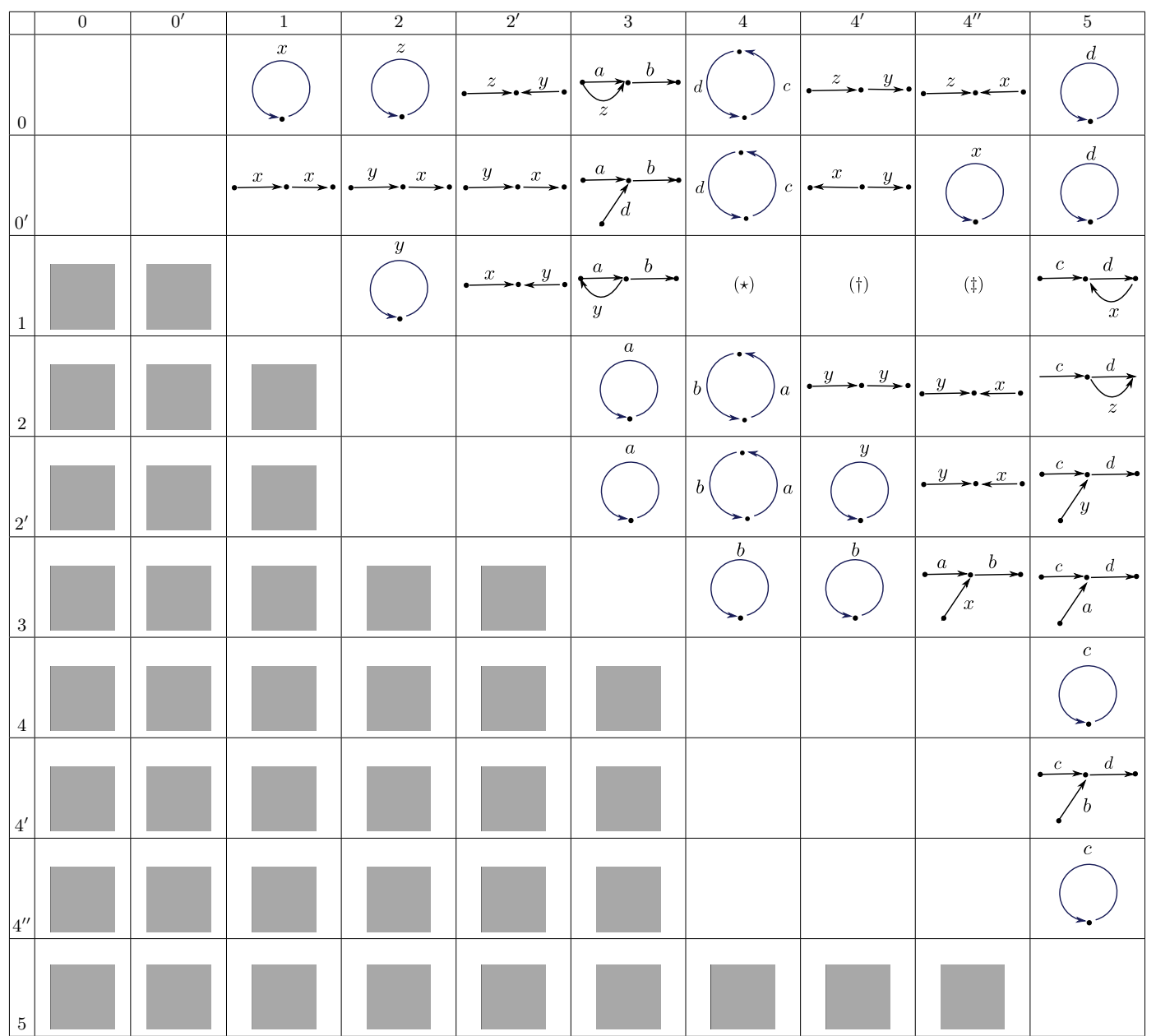

Fig. 5. Identifying two vertices tagged by distinct integers creates persistent patterns. 
This is indeed a back pattern because its vertices are pairwise distinct thanks to Lemma 3 .

We treat the case where the hypothesis $c d \geq y z$ was used to derive $G \succ_{\mathcal{K}} H$ in a similar way. In this case, the following back pattern appears:

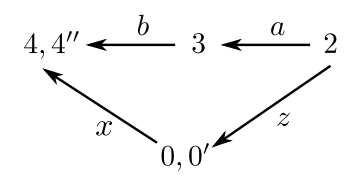

This concludes the proof of this lemma.

Now we can complete the proof of Prop. 2

Proof (of the second part of Prop. 2). Let us show that $\mathcal{G}(\eta){ }_{\mathcal{K}} \mathcal{G}(\rho)$. Suppose for contradiction that $\mathcal{G}(\eta) \boldsymbol{}_{\mathcal{K}} \mathcal{G}(\rho)$. Note that $\mathcal{G}(\eta)$ does not contain a persistent pattern and that $\mathcal{G}(\eta)$ is homomorphic to itself, hence by Lem. 4 , we have that $\mathcal{G}(\eta) \triangleright \mathcal{G}(\rho)$, which is clearly not possible because the edges of $\mathcal{G}(\eta)$ labeled by $a$ cannot be preserved by such a homomorphism. 


\section{References}

1. H. Andréka and D. Bredikhin. The equational theory of union-free algebras of relations. 33(4):516-532, 1995. doi:10.1007/BF01225472.

2. H. Andréka and S. Mikulás. Axiomatizability of positive algebras of binary relations. Algebra universalis, 66(7), 2011. An erratum appears at http://www.dcs.bbk.ac uk/ szabolcs/AM-AU-err6.pdf doi:10.1007/s00012-011-0142-3

3. E. Cosme-Llópez and D. Pous. K4-free graphs as a free algebra. In K. G. Larsen, H. L. Bodlaender, and J. Raskin, editors, 42nd International Symposium on Mathematical Foundations of Computer Science, MFCS 2017, August 21-25, 2017 - Aalborg, Denmark, volume 83 of LIPIcs, pages 76:1-76:14. Schloss Dagstuhl - LeibnizZentrum für Informatik, 2017. doi:10.4230/LIPIcs.MFCS.2017.76

4. A. Doumane. Graph characterization of the universal theory of relations. working paper or preprint, May 2021. URL: https://hal.archives-ouvertes.fr/ hal-03226221, doi:10.4230/LIPIcs.MFCS.2021.11.

5. A. Doumane and D. Pous. Non axiomatisability of positive relation algebras with constants, via graph homomorphisms. In I. Konnov and L. Kovács, editors, 31st International Conference on Concurrency Theory, CONCUR 2020, September 1-4, 2020, Vienna, Austria (Virtual Conference), volume 171 of LIPIcs, pages 29:1-29:16. Schloss Dagstuhl - Leibniz-Zentrum für Informatik, 2020. doi:10.4230/LIPIcs CONCUR . 2020.29

6. P. Freyd and A. Scedrov. Categories, Allegories. 1990.

7. R. Hirsch and I. Hodkinson. Relation algebras by games. Bulletin of Symbolic Logic, 9(4):515-520, 2003.

8. R. Hirsch and S. Mikulas. Representable semilattice-ordered monoids. Algebra universalis, 6, 2007. doi:DOI:10.1007/s00012-007-2055-8.

9. S. Mikulás. URL: http://www.dcs.bbk.ac.uk/ szabolcs/AM-AU-err6.pdf

10. D. Monk. On representable relation algebras. The Michigan mathematical journal, 31(3):207-210, 1964. doi:10.1307/mmj/1028999131.

11. A. Tarski. On the calculus of relations. J. of Symbolic Logic, 6(3):73-89, 1941.

12. A. Tarski. Contributions to the theory of models. iii. 1954. 